\title{
Spectral Efficiency of Variable Density Cellular Systems with Realistic System Models
}

\author{
Symeon Chatzinotas, Muhammad Ali Imran, Costas Tzaras \\ Centre for Communication Systems Research \\ University of Surrey, United Kingdom, GU2 7XH \\ Email: \{S.Chatzinotas, M.Imran, C.Tzaras\}@ surrey.ac.uk
}

\begin{abstract}
In the information-theoretic literature, multicell joint processing has been shown to produce high spectral efficiencies. However, the majority of existing results employ simplified models and normalized variables and in addition they consider only the sum-rate capacity, neglecting the individual user rates. In this paper, we investigate a realistic cellular model which incorporates flat fading, path loss and distributed users. Furthermore, the presented results are produced by varying the cell density of the cellular system, while practical values are used for system parameters, such as users per cell, transmitted power, path loss exponent. What is more, we study the effect of sumrate maximization on the fairness of user rate distribution by comparing channel-dependent and random user orderings within the joint encoding/decoding process.
\end{abstract}

\section{INTRODUCTION}

During the last decades, wireless cellular systems have been through an intensive evolutionary process, moving from analog voice streams to digital data services. As the demand for cost-efficient high-rate wireless services increases, the wireless network operators have to employ new wireless architectures in order to achieve higher data-rates. However, in spite of the evolution of the wireless cellular technologies, the increase in the system complexity becomes disproportional with respect to the provided spectral efficiency gain. Therefore, the research community as well as the industrial sectors have begun a quest for alternative cellular architectures which have the ability to provide high spectral efficiencies. In this direction, cooperative wireless cellular architectures, such as Base Station (BS) cooperation, are gaining momentum as a dominant candidate for an alternative approach in wireless cellular networks.

In the research area of the BS cooperation a.k.a. multicell joint processing, the first concrete result for the single antenna case was presented by Wyner in [1]. Using a very simple but tractable model for the cellular uplink channel, Wyner showed the importance of joint decoding at the Base Station (BS) receivers (hyper-receiver) and found closed forms for the maximum sum-rate capacity under the assumption of multicell joint decoding. This model triggered the interest of the research community in the cellular capacity limits and it was subsequently extended for flat fading environments [2]. In addition, [3] has investigated the capacity scaling in the asymptotic regime where the number of BS antennas and the number of User Terminals (UTs) grow large. One major assumption shared amongst the aforementioned models is that the cell density is fixed and only physically adjacent cells interfere. Letzepis in [4], extended the Wyner's model by assuming multiple-tier interference and incorporated a distance-dependent path loss factor in order to study the effect of cell density in a variable cell-density linear cellular array. However, the assumption of user collocation was still maintained to keep the model tractable. In this paper, we extend the aforementioned models by considering a cellular system which encompasses a number of realistic properties, namely power-law path loss, multiple-tier interference and variable cell density. Most importantly, the considered model employs the hexagonal planar pattern of cells and it incorporates a realistic assumption which has been missing from the previous models, i.e. uniform distribution of UTs over the planar coverage area. Based on the described model, the focus of this paper is to study the sum-rate and user-rate spectral efficiency of both uplink and downlink cellular channels. More specifically, the outline of the paper is as follows. Section II describes the considered cellular model and justifies the employed parameters. Section III evaluates the sum-rate spectral efficiency of the cellular system in both uplink and downlink channel. Section IV focuses on the probability distribution of the individual rates and investigates the effect of sum-rate maximization on the fairness of the cellular system. Finally, Section $\mathrm{V}$ provides a conclusive discussion of the presented findings.

\section{Channel Model Description}

\section{A. Uplink Cellular Channel}

Assume that $K$ users are uniformly distributed over a planar cellular array comprising $N$ BSs (Figure 1). Each UT and BS is equipped with one omnidirectional antenna. Assuming flat fading the received signal at $\mathrm{BS} n$, at time index $i$, will be given by:

$$
y^{n}[i]=\sum_{j=1}^{N} \sum_{k=1}^{K} \varsigma_{k}^{j n} g_{k}^{j n}[i] x_{k}^{j}[i]+z^{n}[i]
$$

where $x_{k}^{j}[i]$ is the $i$ th complex channel symbol transmitted by the $k$ th UT of the $j$ th cell and $\left\{g_{k}^{n j}\right\}$ are independent, strictly stationary and ergodic complex random processes in the time index $i$, which represent the flat fading processes experienced in the transmission path between the $j$ th BS and the $k$ th UT in the $n$th cell. The fading coefficients are assumed to have unit power, i.e. $\mathbb{E}\left[\left|g_{k}^{n j}[i]\right|^{2}\right]=1$ for all $(n, j, k)$. The 
path-loss coefficients $\varsigma_{k}^{n j}$ in the transmission path between the $j$ th BS and the $k$ th UT in the $n$th cell are calculated according to the "modified" power-law path loss model [4], [5]: $\varsigma_{k}^{n j}=\left(1+d_{k}^{n j}\right)^{-\eta / 2}$, where $d_{k}^{n j}$ is the distance between the $j$ th BS and the $k$ th UT in the $n$th cell and $\eta$ is the pathloss exponent. All UTs are subject to an individual power constraint, i.e. $\mathbb{E}\left[\left|x_{k}^{j}[i]\right|^{2}\right] \leq P$ for all $(k, j)$. The model can be more compactly expressed as a vector memoryless channel of the form (dropping the time index $i$ ):

$$
\mathbf{y}=\mathbf{H x}+\mathbf{z}
$$

where the vector $\mathbf{y}=\left[y^{1} \ldots y^{N}\right]^{T}$ represents received signals by the BSs, the vector $\mathbf{x}=\left[\begin{array}{lll}x_{1}^{1} \ldots & \ldots & x_{K}^{N}\end{array}\right]^{T}$ represents transmit signals by all the UTs of the cellular system and the components of vector $\mathbf{z}=\left[\begin{array}{lll}z^{1} \ldots & z^{N}\end{array}\right]^{T}$ are independent identically distributed (i.i.d.) complex circularly symmetric (c.c.s.) random variables representing Additive White Gaussian Noise (AWGN) with $\mathbb{E}\left[z^{n}\right]=0, \mathbb{E}\left[\left|z^{n}\right|^{2}\right]=\sigma^{2}$.

\section{B. Downlink Cellular Channel}

Assuming perfect channel reciprocity, the received signal at UT $k=1 \ldots K$ of cell $n=1 \ldots N$, at time index $i$, is:

$$
\tilde{y}_{k}^{n}[i]=\sum_{j=1}^{N} \varsigma_{k}^{n j} g_{k}^{n j}[i] \tilde{x}^{j}[i]+\tilde{z}_{k}^{n}[i]
$$

where $\tilde{x}^{j}[i]$ is the complex signal transmitted by BS $j=$ $1 \ldots N$ at the $i$ th time instant. All BSs are subject to a sum power constraint, i.e. $\mathbb{E}\left[\sum_{j=1}^{N}\left|\tilde{x}^{j}[i]\right|^{2}\right] \leq P_{\text {tot }}$. The model can be written in vector form as:

$$
\tilde{\mathbf{y}}=\tilde{\mathbf{H}} \tilde{\mathbf{x}}+\tilde{\mathbf{z}}
$$

where the vector $\tilde{\mathbf{y}}=\left[\tilde{y}_{1}^{1} \ldots \tilde{y}_{K}^{N}\right]^{T}$ represents received signals by all the UTs of the cellular system, the vector $\tilde{\mathbf{x}}=$ $\left[\tilde{x}^{1} \ldots \tilde{x}^{N}\right]^{T}$ represents transmit signals by the BSs and the components of vector $\tilde{\mathbf{z}}=\left[\tilde{z}_{1}^{1} \ldots \tilde{z}_{K}^{N}\right]^{T}$ are i.i.d. c.c.s. random variables representing AWGN with $\mathbb{E}\left[\tilde{z}_{k}^{n}\right]=0, \mathbb{E}\left[\left|\tilde{z}_{k}^{n}\right|^{2}\right]=\sigma^{2}$.

\section{Channel Matrix}

The channel matrix $\mathbf{H}$ of the uplink channel can be written as $\mathbf{H}=\boldsymbol{\Sigma} \odot \mathbf{G}$, where $\odot$ denotes Hadammard multiplication, $\boldsymbol{\Sigma}$ is a $N \times K N$ deterministic matrix, comprising the pathloss coefficients and $\mathbf{G}$ is a Gaussian $N \times K N$ matrix with i.i.d. c.c.s. elements of variance 1 , comprising the fading coefficients. Based on the "modified" power-law path loss model, the entries of the $\boldsymbol{\Sigma}$ matrix can be defined by the variance profile function

$$
\varsigma(u, v)=(1+d(u, v))^{-\eta / 2}
$$

where $u \in[0,1]$ and $v \in[0, K]$ are the normalized indexes for the UTs and the BSs respectively and $d(u, v)$ is the normalized distance between BS $u$ and UT $v$. On the grounds of channel reciprocity, the channel matrix of the downlink channel can be written as $\tilde{\mathbf{H}}=\mathbf{H}^{\dagger}=\boldsymbol{\Sigma}^{\dagger} \odot \mathbf{G}^{\dagger}$.

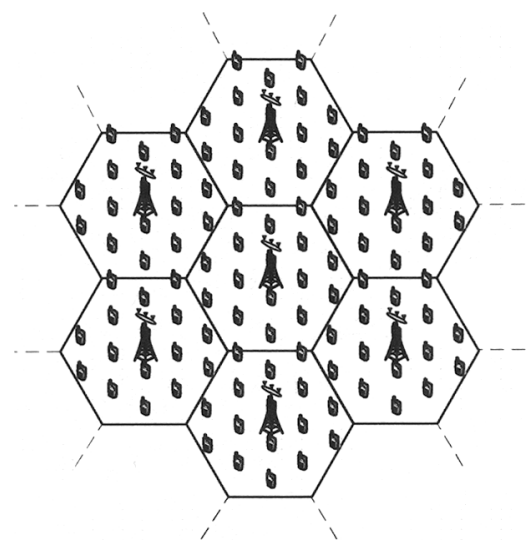

Fig. 1. Ground plan of the cellular system comprising of UTs positioned on a uniform planar regular grid.

\section{Sum-Rate SPECTRAl EfFiciency}

\section{A. Uplink Spectral Efficiency}

In the uplink channel it has been shown that the capacity region can be achieved by employing Superposition Coding (SP) in combination with Successive Interference Cancellation (SIC). Considering this coding scheme, the asymptotic sumrate spectral efficiency $C_{\text {opt }}$ for this model is given by [6]

$$
\begin{aligned}
C_{\mathrm{opt}} & =\lim _{N \rightarrow \infty} \frac{1}{N} \mathcal{I}(\mathbf{x} ; \mathbf{y} \mid \mathbf{H}) \\
& =\lim _{N \rightarrow \infty} \mathbb{E}\left[\frac{1}{\mathrm{~N}} \sum_{\mathrm{i}=1}^{\mathrm{N}} \log \left(1+\frac{\hat{\gamma}}{K} \lambda_{i}\left(\frac{1}{N} \mathbf{H H}^{\dagger}\right)\right)\right] \\
& =\int_{0}^{\infty} \log \left(1+\frac{\hat{\gamma}}{K} x\right) d \mathrm{~F}_{\frac{1}{N} \mathbf{H} \mathbf{H}^{\dagger}}(x) \\
& =\mathcal{V}_{\frac{1}{N} \mathbf{H} \mathbf{H}^{\dagger}}(\hat{\gamma} / K)=K \mathcal{V}_{\frac{1}{N} \mathbf{H}^{\dagger} \mathbf{H}^{\prime}}(\hat{\gamma} / K)
\end{aligned}
$$

where $\hat{\gamma}=K N \gamma$ and $\gamma=P / \sigma^{2}$ is the system- and UTtransmit power normalized with the receiver noise power respectively, $\lambda_{i}(\mathbf{X})$ are the eigenvalues of matrix $\mathbf{X}$ and $\mathcal{V}_{\mathbf{X}}$ is the Shannon transform [6] of a random square Hermitian matrix $\mathbf{X}$. For $\mathbf{G} \sim \mathcal{C N}\left(\mathbf{0}, \mathbf{I}_{N}\right)$, the empirical eigenvalue distribution of $\frac{1}{N} \mathbf{G}^{\dagger} \mathbf{G}$ converges almost surely (a.s.) to the nonrandom limiting eigenvalue distribution of the MarčenkoPastur law, whose Shannon transform is given by

$$
\mathcal{V}_{\frac{1}{N} \mathbf{G}^{\dagger} \mathbf{G}}(y) \stackrel{\text { a.s. }}{\longrightarrow} \mathcal{V}_{\mathrm{MP}}(y, K)
$$

$$
\begin{aligned}
& \text { where } \quad \mathcal{V}_{\mathrm{MP}}(y, K)=\log \left(1+y-\frac{1}{4} \phi(y, K)\right) \\
& +\frac{1}{K} \log \left(1+y K-\frac{1}{4} \phi(y, K)\right)-\frac{1}{4 K y} \phi(y, K)
\end{aligned}
$$

and $\phi(y, K)=$

$$
\left(\sqrt{y(1+\sqrt{K})^{2}+1}-\sqrt{y(1-\sqrt{K})^{2}+1}\right)^{2} .
$$


According to the Free Probability approach in [4], [7], if $\boldsymbol{\Sigma}$ is a path loss dependent $N \times K N$ deterministic matrix, the limiting eigenvalue distribution of $\mathbf{H}^{\dagger} \mathbf{H}$ and its Shannon transform is approximated by a scaled version of the Marčenko-Pastur law

$$
\begin{gathered}
\mathcal{V}_{\frac{1}{N} \mathbf{H}^{\dagger} \mathbf{H}}(\hat{\gamma} / K) \simeq \mathcal{V}_{\mathrm{MP}}\left(q_{K}(\boldsymbol{\Sigma}) \hat{\gamma} / K, K\right) \\
\text { where } q_{K}(\boldsymbol{\Sigma}) \triangleq\|\boldsymbol{\Sigma}\|^{2} / K N^{2}
\end{gathered}
$$

with $\|\boldsymbol{\Sigma}\| \triangleq \sqrt{\operatorname{tr}\left\{\boldsymbol{\Sigma}^{\dagger} \boldsymbol{\Sigma}\right\}}$ being the Frobenius norm of the $\boldsymbol{\Sigma}$ matrix. In the asymptotic case $q_{K}(\boldsymbol{\Sigma})$ is given by

$$
\lim _{N \rightarrow \infty} q_{K}(\boldsymbol{\Sigma})=\frac{1}{K} \int_{0}^{1} \int_{0}^{K} \varsigma^{2}(u, v) d u d v .
$$

Since the variance profile function defines a rectangular blockcirculant matrix with $1 \times K$ blocks which is symmetric about $u=K v$ for a toric planar cellular model , the channel matrix $\mathbf{H}$ is asymptotically row-regular [6, Definition 2.10] and thus previous equation can be simplified to

$$
\lim _{N \rightarrow \infty} q_{K}(\boldsymbol{\Sigma})=\frac{1}{K} \int_{0}^{K} \varsigma^{2}(v) d v, \forall u \in[0,1] .
$$

According to [4], this approximation holds for UTs collocated with the BS in a linear cellular array. In [8], [7] we show that this approximation also holds for the case where the UTs are distributed over a planar cellular array. In order to calculate the uplink spectral efficiency analytically, a closed form for the variance profile function is needed for calculating $q_{K}(\boldsymbol{\Sigma})$ in Equation (13). Based on the analysis in [8], the variance profile function for a planar cellular array is given by

$$
\begin{aligned}
\varsigma(v) & =\varsigma_{\mathrm{CO}}(v) \sqcap\left(\frac{N t-\frac{K}{2}}{K}\right) \\
& +\sum_{m=1}^{M} \varsigma_{\mathrm{I}}^{m}\left(N t-K-\sum_{c=1}^{m-1} 6 c K\right) \\
& \sqcap\left(\frac{N t-K-\left(\sum_{c=1}^{m-1} 6 c K+3 m K\right)}{6 m K}\right)
\end{aligned}
$$

The rect functions denoted by $\sqcap$ are used in order to apply different variance profile functions $\left(\varsigma_{\mathrm{CO}}, \varsigma_{\mathrm{I}}^{m}\right)$ to UTs that belong to the cell of interest and to each of the $M$ interfering tiers. The factor $6 m K$ is due to the fact that the $m$ th interfering tier includes $6 m$ cells and thus $6 m K$ UTs, which can be treated equally on the grounds of symmetry. The reader is referred to [8] for more details on Equation (14).

\section{B. Downlink Spectral Efficiency}

In order to evaluate the optimal downlink sum-rate spectral efficiency, the problem of power allocation has to be solved. Power allocation determines how the available power $P_{t o t}$ is distributed among the UTs. The aim is to find the power allocation strategy which maximizes the sum-rate spectral efficiency of the cellular system at each time instance. Considering that capacity region of the downlink cellular channel is achieved by the Dirty Paper Coding (DPC) scheme [9], this objective can be translated to the following problem:

maximize

$$
\begin{aligned}
\mathrm{C}_{\mathrm{DL}}\left(\mathbf{H}, P_{t o t}\right) & =\sum_{i=1}^{K N} \log \frac{\operatorname{det}\left(\mathbf{I}+\mathbf{h}_{i}^{\dagger}\left(\sum_{j=1}^{i} \boldsymbol{\Gamma}_{j}\right) \mathbf{h}_{i}\right)}{\operatorname{det}\left(\mathbf{I}+\mathbf{h}_{i}^{\dagger}\left(\sum_{j=1}^{i-1} \boldsymbol{\Gamma}_{j}\right) \mathbf{h}_{i}\right)} \\
\text { subject to } & \sum_{i=1}^{K N} \operatorname{Tr}\left(\boldsymbol{\Gamma}_{i}\right) \leq P_{t o t} \text { and } \boldsymbol{\Gamma}_{i} \geq 0
\end{aligned}
$$

where $\boldsymbol{\Gamma}_{i} \in \mathbb{C}^{N \times N}$ are the downlink input covariance matrices and $\mathbf{h}_{i} \in \mathbb{C}^{1 \times N}$ are the column vectors of the channel matrix $\mathbf{H}$, namely the channel gains of UT $i$ w.r.t. $N$ BSs. As it can be seen, Equation (15) is neither a concave nor a convex function of $\Gamma_{i}$ and thus the entire space of covariance matrices should be examined to meet the objective [10], [11]. However, this obstacle can be overcome by utilizing the principles of duality. More specifically, instead of maximizing Equation (15), the following dual maximization problem can be considered:

maximize

$$
\begin{aligned}
\mathrm{C}_{\mathrm{UL}}\left(\mathbf{H}^{\dagger}, P_{\text {tot }}\right)= & \log \operatorname{det}\left(\mathbf{I}+\sum_{i=1}^{K N} \mathbf{h}_{i} \mathbf{Q}_{i} \mathbf{h}_{i}^{\dagger}\right) \\
\text { subject to } & \sum_{i=1}^{K N} \operatorname{Tr}\left(\mathbf{Q}_{i}\right) \leq P_{\text {tot }} \text { and } \mathbf{Q}_{i} \geq 0
\end{aligned}
$$

where $\mathbf{Q}_{i} \in \mathbb{C}$ are the uplink input covariance matrices, namely the transmit power of each UT. It should be noted that in the single-antenna case $\mathbf{Q}_{i}$ reduces to a scalar $q_{i}$ and thus the power constraint can be rewritten as $\sum_{i=1}^{K N} q_{i} \leq P_{t o t}$. Equation (17) is a convex function of $\mathbf{Q}_{i}$ [12] and hence a number of algorithms have been proposed in order to solve this convex optimization problem, i.e. the steepest descend method [13], the dual decomposition method [14] and the iterative waterfilling method [11]. The algorithm in [15] addresses the per-BS constrained problem, which is a more realistic assumption for cellular systems. However, this algorithm produces same spectral efficiency results as the per-system constrained algorithms in high SNR conditions and in addition it has a higher complexity. On these grounds, algorithms [11] and [14] were utilized to produce the results of this paper.

\section{Realistic Parameters and Results}

In order to apply the aforementioned results to real-world cellular systems, a reference distance $d_{0}$ is required to interconnect the actual distance $\hat{d}(t)$ and the normalized distance $d(t)=\hat{d}(t) / d_{0}$. It should be noted that the actual distance $\hat{d}(t)$ is not measured w.r.t. the BS position, but w.r.t. a circle of radius $d_{0}$ around the BS position. If the power loss at the reference distance $d_{0}$ is $L_{0}$, the scaled variance profile function is given by

$$
\varsigma(d(t))=\sqrt{L_{0}\left(1+\hat{d}(t) / d_{0}\right)^{-\eta}} .
$$


TABLE I

PARAMETERS FOR PRACTICAL CELLULAR SYSTEMS

\begin{tabular}{c|c|l} 
Parameter & Symbol & Value/Range (units) \\
\hline Cell Radius & $R$ & $0.1-3 \mathrm{Km}$ \\
Reference Distance & $d_{0}$ & $1 \mathrm{~m}$ \\
UTs per Km ${ }^{2}$ & $K$ & 16 \\
UT Transmit Power & $P_{T}$ & $200 \mathrm{~mW}$ \\
BS Transmit Power & $P_{B S}$ & $50 \mathrm{~W}$ \\
Thermal Noise Density & $N_{0}$ & $-169 \mathrm{dBm} / \mathrm{Hz}$ \\
Channel Bandwidth & $B$ & $5 \mathrm{MHz}$ \\
\hline
\end{tabular}

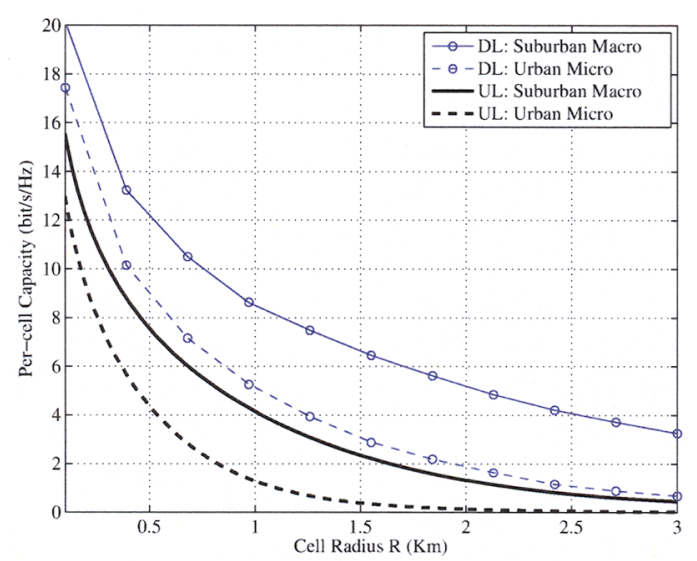

Fig. 2. Practical sum-rate spectral efficiency results for the uplink and the downlink channel of a joint-processing cellular system.

The values of $L_{0}$ and $d_{0}$ have been fitted to the three scenarios presented in [16] as follows: a) "Suburban Macro" $L_{0}=$ $31.5 d B, \eta=3.5$, b) "Urban Macro" $L_{0}=34.5 d B, \eta=3.5$ and "Urban Micro" $L_{0}=34.53 d B, \eta=3.8$. The presented results refer to the per-cell sum-rate spectral efficiency averaged over a large number of fading realizations $g_{k}^{n m}$ and UT positions $d_{k}^{n m}$ and they are plotted vs. a variable cell radius $R$ using the parameters of Table I. As it can be seen, higher per-cell capacities can be achieved in the downlink channel in comparison to the uplink channel due to the laxer power constraint and the high-power transmit capabilities of the BSs. However, the spectral efficiency gap closes as the number of UTs per $\mathrm{Km}^{2}$ increases, since the total received power in the uplink increases, while the transmit power in the downlink remains unchanged.

\section{INDIVIDUAL RATES AND FAIRNESS}

In order to determine the individual rate of each UT, a UT ordering is needed for the encoding and decoding of signals in the downlink and uplink channel respectively. More specifically, if $i=[1,2, \ldots, K N]$ is a vector containing the ordered UT indexes, then the UT ordering $\pi(i)$ denotes a permutation of this vector. Let us now consider an arbitrary UT ordering in order to observe its effect of the individual rates. In the DPC encoding of the downlink channel, the first-encoded UT signals are less favoured than the last-encoded UT signals, since the encoder is still unaware of the interference and it is unable to remove it. Similarly, in the SIC decoding of the uplink channel, the first-decoded UT signals are less favoured than the last-decoded UT signals, since the decoder has not still decoded and removed the interference. In this context, the effect of the following UT orderings on the individual UT rates is considered:

- Sum Channel Gain ordering: the UT ordering is determined by the norm of the instant channel matrix of each UT w.r.t. the $N$ BSs, i.e. $\left\|\mathbf{h}_{i}\right\|=\sum_{j=1}^{N}\left(h_{i}\right)_{j}^{2}$. Two subcases can be considered: "Increasing Gain" and "Decreasing Gain" ordering.

- Random ordering: the UT ordering is totally random.

\section{A. Uplink Individual Rates}

Considering a SIC decoding order $\pi(i)$ and its reverse $\tilde{\pi}(i)$ produced by flipping $\pi(i)$, the individual rate of the $j^{t h}$ UT is given by:

$$
C_{\mathrm{UL}}^{\tilde{\pi}(i)}=\mathbb{E}\left\{\log \operatorname{det}\left(\frac{\mathbf{I}+\gamma \sum_{j=1}^{i} \mathbf{h}_{\tilde{\pi}(j)} \mathbf{h}_{\tilde{\pi}(j)}^{\dagger}}{\mathbf{I}+\gamma \sum_{j=1}^{i-\mathbf{1}} \mathbf{h}_{\tilde{\pi}(j)} \mathbf{h}_{\tilde{\pi}(j)}^{\dagger}}\right)\right\}
$$

It is worth noticing that the uplink individual rates are affected by multiple UT channel matrices $h$, which is due to the nature of the MAC channel. Figure 3 depicts the Cumulative Distribution of the individual uplink rates. As it can be seen, the Random UT ordering produces a very unfair rate distribution, where only $13 \%$ of the UTs take advantage of the high sum-rate spectral efficiency produced by the multicell joint decoding (higher than $2 \mathrm{bits} / \mathrm{sec} / \mathrm{Hz}$ ). Increasing and Decreasing Gain UT orderings provide a slightly fairer distribution, but the majority of UTs are still deficiently served, since the lack of power control gives rise to the near-far effect.

\section{B. Downlink Individual Rates}

After solving the uplink optimization problem of Equation (17), the downlink covariance matrices $\Gamma$ can be calculated given the uplink covariance matrices $\mathbf{Q}$ and the UT ordering, based on the MAC-to-BC transformation described in [10]. Knowing the downlink covariance matrices and considering a DPC encoding order $\pi(i)$, the individual downlink rates can be calculated using the following formula:

$$
\mathrm{C}_{\mathrm{DL}}^{\pi(i)}=\mathbb{E}\left\{\log \operatorname{det}\left(\frac{\mathbf{I}+\mathbf{h}_{\pi(i)}^{\dagger}\left(\sum_{j=1}^{i} \boldsymbol{\Gamma}_{\pi(j)}\right) \mathbf{h}_{\pi(i)}}{\mathbf{I}+\mathbf{h}_{\pi(i)}^{\dagger}\left(\sum_{j=1}^{i-1} \boldsymbol{\Gamma}_{\pi(j)}\right) \mathbf{h}_{\pi(i)}}\right)\right\}
$$

In this point, it is worth mentioning that in the downlink channel the individual rates are affected not only by the UT ordering, but also by the power allocation strategy. Furthermore, the downlink individual rates are affected by a single UT channel matrix $\mathbf{h}$, which is due to the nature of the $\mathrm{BC}$ channel. Figure 4 depicts the Cumulative Distribution of the individual downlink rates. As it can be seen, the Increasing Gain UT ordering produces a very unfair rate distribution, where only $14 \%$ of the UTs take advantage of the high sum-rate spectral efficiency produced by the multicell joint encoding. Random and Decreasing Gain UT orderings provide a more fair distribution, where $40 \%$ of the UTs achieve rates higher than 2 bits/sec/Hz. However, there are still UTs which 


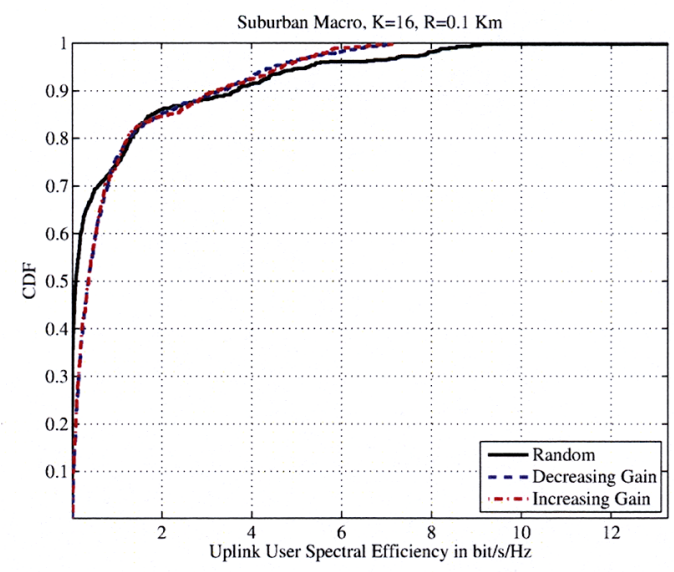

Fig. 3. Cumulative Distribution of the user spectral efficiency (bits/sec/Hz) for the uplink channel. Parameters: $R=0.1 \mathrm{Km}$.

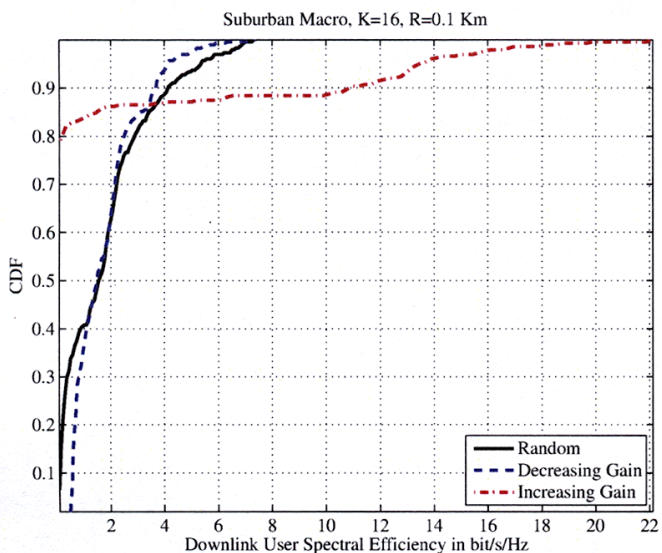

Fig. 4. Cumulative Distribution Function of the user spectral efficiency (bits/sec/Hz) for the downlink channel. Parameters: $R=0.1 \mathrm{Km}$.

are deficiently served, since the iterative waterfilling power allocation favours the cell-centre UTs that are more probable to have a strong channel gain.

\section{DISCUSSION AND CONCLUSION}

In this paper, we have investigated a realistic cellular model which incorporates Rayleigh flat fading, power-law path loss and uniformly distributed UTs. In this context, high sum-rate spectral efficiencies can be achieved (up to $25 \mathrm{bits} / \mathrm{sec} / \mathrm{Hz}$ depending on the cell radius), but the distribution of the individual rates seems to be rather unfair. In order to achieve the fairest possible distribution, the space of all possible UT orderings has to be searched in order to identify the ordering that maximizes the minimum rate at each time instant. An algorithm to tackle this minmax problem for the downlink channel has been presented in [17]. However, the complexity of this problem makes it prohibitive for practical systems. In this direction, we have shown that this unfairness can be mitigated by employing the appropriate UT ordering. As future work, more sophisticated power control and allocation algorithms will be considered in order to optimize the system performance w.r.t. multiple objectives e.g. sum-rate capacity maximization and fair user rate distribution.

\section{ACKNOWLEDGMENT}

The work reported in this paper has formed part of the "Fundamental Limits to Wireless Network Capacity" Elective Research Programme of the Virtual Centre of Excellence in Mobile \& Personal Communications, Mobile VCE, www.mobilevce.com. This research has been funded by the following Industrial Companies who are Members of Mobile VCE - BBC, BT, Huawei, Nokia, Nokia Siemens Networks, Nortel, Vodafone. Fully detailed technical reports on this research are available to staff from these Industrial Members of Mobile VCE. The authors would like to thank Prof. G. Caire and Prof. D. Tse for the useful discussions.

\section{REFERENCES}

[1] A. Wyner, "Shannon-theoretic approach to a Gaussian cellular multipleaccess channel," IEEE Trans. Inform. Theory, vol. 40, no. 6, pp. 17131727, Nov. 1994

[2] O. Somekh and S. Shamai, "Shannon-theoretic approach to a Gaussian cellular multiple-access channel with fading," IEEE Trans. Inform. Theory, vol. 46, no. 4, pp. 1401-1425, Jul 2000.

[3] D. Aktas, M. Bacha, J. Evans, and S. Hanly, "Scaling results on the sum capacity of cellular networks with MIMO links," IEEE Trans. Inform. Theory, vol. 52, no. 7, pp. 3264-3274, July 2006.

[4] N. A. Letzepis, "Gaussian cellular multiple access channels," Ph.D. dissertation, Institute for Telecommunications Research, University of South Australia, Dec 2005.

[5] L. Ong and M. Motani, "On the capacity of the single source multiple relay single destination mesh network," Ad Hoc Netw., vol. 5, no. 6, pp. 786-800, 2007.

[6] A. M. Tulino and S. Verdu, "Random matrix theory and wireless communications," Commun. Inf. Theory, vol. 1, no. 1, pp. 1-182, 2004

[7] S. Chatzinotas, M. Imran, and C. Tzaras, "On the capacity of variable density cellular systems under multicell decoding," IEEE Communications Letters, vol. 12, no. 7, Jul 2008.

[8] - " "Optimal information theoretic capacity of planar cellular uplink channel," in Signal Processing Advances in Wireless Communications, 9th IEEE International Workshop on (SPAWC'08), Jul 2008.

[9] H. Weingarten, Y. Steinberg, and S. Shamai, "The capacity region of the Gaussian multiple-input multiple-output broadcast channel," IEEE Trans. Inform. Theory, vol. 52, no. 9, pp. 3936-3964, 2006.

[10] S. Vishwanath, N. Jindal, and A. Goldsmith, "Duality, achievable rates, and sum-rate capacity of Gaussian MIMO broadcast channels," IEEE Trans. Inform. Theory, vol. 49, no. 10, pp. 2658-2668, 2003.

[11] N. Jindal, W. Rhee, S. Vishwanath, S. Jafar, and A. Goldsmith, "Sum power iterative water-filling for multi-antenna gaussian broadcast channels," IEEE Trans. Inform. Theory, vol. 51, no. 4, pp. 1570-1580, Apr 2005.

[12] W. Yu, W. Rhee, S. Boyd, and J. Cioffi, "Iterative water-filling for Gaussian vector multiple-access channels," IEEE Trans. Inform. Theory, vol. 50, no. 1, pp. 145-152, 2004.

[13] H. Viswanathan, S. Venkatesan, and H. Huang, "Downlink capacity evaluation of cellular networks with known-interference cancellation," IEEE J. Select. Areas Commun., vol. 21, no. 5, pp. 802-811, 2003.

[14] W. Yu, "Sum-capacity computation for the Gaussian vector broadcast channel via dual decomposition." IEEE Trans. Inform. Theory, vol. 52, no. 2, pp. 754-759, 2006.

[15] T. Lan and W. Yu, "Input optimization for multi-antenna broadcast channels with per-antenna power constraints," in IEEE Global Telecommunications Conference (GLOBECOM '04), vol. 1, 2004, pp. 420-424.

[16] ETSI TR 125996 V7.0.0, "Universal mobile telecommunications system (UMTS); spatial channel model for multiple input multiple output (MIMO) simulations (3GPP TR 25.996 ver. 7.0.0 rel. 7)," Jun 2007.

[17] J. Lee and N. Jindal, "Symmetric capacity of MIMO downlink channels," Information Theory, 2006 IEEE International Symposium on, pp. 10311035, July 2006. 